\title{
Simulation of Transient Temperature Field in the Selective Laser Sintering Process of W/Ni Powder Mixture
}

\author{
Jiwen Ren, Jianshu Liu, and Jinju Yin \\ The School of Electromechanical Engineering, East China Jiaotong University, \\ Nanchang, 330013, P.R. China \\ renjiwena163. com
}

\begin{abstract}
Selective laser sintering (SLS) is an attractive rapid prototyping and manufacturing (RP\&M) technology as well as two-component metal powder has high melting pointer, high mechanical properties and high wear resistance. Hence, it's meaningful to analyze its temperature field distribution and dynamical evolution rule in sintering process. A three-dimensional transient finite element model of SLS on the two-component metal powder W/Ni has been developed to predict the temperature field distribution in this paper. The dynamically loading of the moving Gaussian laser thermal resource was realized using the element "birth and death" technology and the ANSYS Parameter Design Language (APDL) in the model. Considering comprehensively thermal convection and the non-linear behavior of material properties etc., the temperature evolution of SLS process has been simulated effectively. The interrelation between the temperature field distribution and the processing parameters are analyzed. The sintering width and depth under certain selected sintering parameters are obtained so as to judge the metallurgical bonding performance between substrate and layers. The result of simulation can provide the theoretical basis for selecting reasonable processing parameters.
\end{abstract}

Keywords: Selective laser sintering, Finite element simulation, Temperature field, Molten pool.

\section{Introduction}

Selective laser sintering(SLS) is an attractive process in the new field of rapid prototyping (RP) which is such an advance manufacturing technology that integrates laser technology, precision machinery, computer-aided design, computer-aided manufacturing, computer numerical control, control technology, and material technology [1]. Direct metal laser sintering (DMLS) is a method of rapid prototyping and manufacturing (RP\&M) which three-dimensional parts are formed directly from metal powders. It includes three major materials such as single-component metal powder, multicomponent metal powder and pre-alloyed powder. Multi-component powder can overcome the shortcomings of the single-component metal powder such as difficult forming, which consists of two metal materials with significantly different melting 
points. The low melting metal powder acts as the bonding phase while the high melting metal powder acts as the framework phase [2]. Research shows that multicomponent metal could reduce plastic deformation in the high temperature and improve mechanical properties of the SLS parts [3]. Therefore, the research on direct laser sintering high melting metal powder has attracted more and more attention.

Nowadays, many scholars at home and abroad work on SLS temperature field simulation [4-6], but there is little research on temperature field simulation of twocomponent metal powder with high melting point. Therefore, it's necessary to systematically analyze the temperature field distribution and the dynamical evolution rules in sintering process of two-component metal powder and carries out accurate simulation and prediction, which can provide the theoretical basis and guidance to experiments of direct laser sintering high melting point metal powder. With the development of computer technology and finite element theory, it has becomes a reasonable way to study the laser sintering temperature field using finite element method (FEM). In this paper, a finite element model for laser sintering has been developed and the load of moving heat source at different times and locations is carried out by ANSYS Parametric Design Language (APDL). At the same time, the formation and evolution of transient temperature field sintering two-component metal powder during SLS and formation mechanism of two-component metal powder has been discussed.

\section{Establishment of Model}

\subsection{Establishment of Finite Element Model}

The finite element model of sintering is shown in Fig. 1. The established finite element model is divided into two parts, namely substrate and powder. The Upper of model is two-component metal powder which is a mixture of $\mathrm{W}$ and $\mathrm{Ni}$ and its size is $3.4 \mathrm{~mm} \times 1.6 \mathrm{~mm} \times 0.2 \mathrm{~mm}$. The substrate material is 45 steel and its size is 4.4 $\mathrm{mm} \times 3 \mathrm{~mm} \times 0.6 \mathrm{~mm}$. In order to ensure the accuracy of the calculation, the sintering powder is meshed by Solid70 with hexahedral and eight-node and the grid size is $0.1 \mathrm{~mm} \times 0.1 \mathrm{~mm} \times 0.1 \mathrm{~mm}$ while the substrate is meshed by Solid90. To save calculation time and improve efficiency, the larger grids are applied to the substrate.

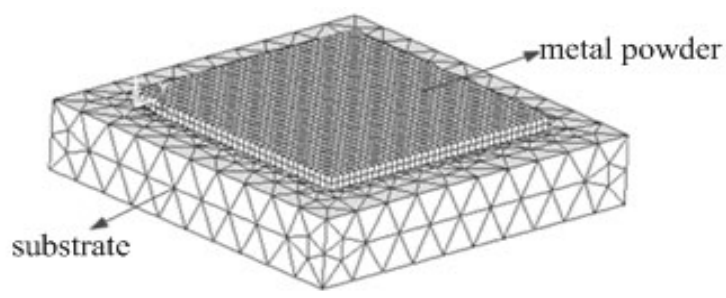

Fig. 1. 3-D finite element model of laser sintering 


\subsection{Initial Conditions and Boundary Conditions}

SLS is a very complex heat treatment process, which is accompanied by three ways of heat transfer including heat conduction, heat convection and heat radiation [7]. Before solving the heat balance equation, we should assume that powder bed has a uniform initial temperature $T_{0}$, which is at room temperature or reaches a certain uniform temperature through preheating, then the initial conditions as follows:

$$
\left.T(x, y, z, t)\right|_{t=0}=T_{0} \text {. }
$$

The convection and radiation between surrounding environment and substrate and the boundaries of powder bed belong to the third type boundary condition, which is expressed as follows.

$$
-\left.k_{e} \frac{\partial T}{\partial z}\right|_{z=0}+h\left(T-T_{E}\right)+\sigma \varepsilon\left(T^{4}-T_{E}^{4}\right)=q .
$$

Where $k_{e}$ is the thermal conductivity of the material, $h$ is convection heat transfer coefficient, $T$ is the temperature of metal powder at a certain moment, $\sigma$ is the Stefan-Boltzmann constant, $T_{E}$ is initial temperature and $\varepsilon$ is thermal emissivity of real object.

\section{Load of Gauss Heat Source}

In the process of sintering, laser energy moving at a certain rate is input to powder bed in the form of heat flux. Generally speaking, the laser energy below $3 \mathrm{kw}$ obeys the Gaussian distribution [8].

$$
q(x, y)=\frac{2 A P}{\pi \omega^{2}} \exp \left(-2 \frac{r^{2}}{\omega^{2}}\right)
$$

Where $q(x, y)$ is laser power density, $\rho$ is laser power, $A$ is absorption rate of powder bed to laser, $r^{2}=\left(x-x_{0}\right)^{2}+\left(y-y_{0}\right)^{2}$ is distance between any point on the powder bed and laser spot center and $\omega$ is radius of laser spot.

In order to make this simulation close to reality, it needs to use the technique of "element birth/death" to exert the heat load at different times at different spots. First, all the element of powder bed is killed in the beginning. Then, the element which needs to be loaded is activated as the sintering goes on, which is used to simulate dynamic sintering process. Next, the heat flux is exerted on the activated element meantime in the meantime the heat flux of last load step should be removed and the 
previous solution results are used as the initial conditions for this solution to calculate thermal conductivity matrix and specific heat matrix. The circle is in turn until the whole sintering process completed.

\section{Thermal Physical Properties of Material}

The sintering powder is obtained by mixing $\mathrm{W}$ powder and $\mathrm{Ni}$ powder according to the percentage of $55 \% \mathrm{Ni}$ and $45 \% \mathrm{~W}$. The material parameters of $\mathrm{Ni}, \mathrm{W}$ and the substrate material of 45 steel are shown $[9,10]$. The equivalent thermal physical parameters of mixture powder such as density and specific heat can be determined by the law of Kopp-Neumann. Among all the thermal physical parameters which have great influence on the performance of powder sintering, the effective thermal conductivity of powder bed is the most important. However, due to the heat transfer mechanism is complex, the precise value is difficult to determine. So the area without sintering cannot be directly defined with thermal physical properties of material, but could be estimated by the following equation [11].

$$
\begin{gathered}
\frac{k_{e}}{k_{g}}=(1-\sqrt{1-\varphi})\left[1+\frac{\varphi k_{r}}{k_{g}}\right]+\sqrt{1-\varphi} \times \\
{\left[\frac{2}{1-\frac{k_{g}}{k_{s}}}\left(\frac{1}{1-\frac{k_{g}}{k_{s}}} \ln \left(\frac{k_{s}}{k_{g}}\right)-1\right)+\frac{k_{r}}{k_{g}}\right] .}
\end{gathered}
$$

Where $k_{g}$ is the thermal conductivity of environment, $k_{s}$ is effective thermal conductivity of solid, $\varphi$ is initial porosity and $k_{r}$ is heat transfer coefficient of powder bed cased by radiation, which can be expressed by the following equation.

$$
k_{r}=4 F \sigma T^{2} D_{p} .
$$

Where $\sigma$ is Stefan-Boltzmann constant, $D_{p}$ is average diameter of powder particles, $T$ is temperature of powder particles and $F$ is apparent coefficient approximately taken as $1 / 3$.

\section{Simulation Results and Discussion}

Fig. 2 shows the scanning pattern of simulation. It includes two layers. Each layer has five scanning paths and the scanning width is $0.3 \mathrm{~mm}$. The other simulation parameters 
include the laser power, the spot diameter, the scanning speed, the thermal absorptivity, the initial temperature and the coefficient of air convection under normal temperature. The values are $300 \mathrm{~W}, 0.4 \mathrm{~mm}, 80 \mathrm{~mm} / \mathrm{s}, 0.3,298 \mathrm{~K}$ and 10 , respectively. In order to master the variation of temperature field in sintering process, some special points on scanning line are selected to analyze, as shown in Fig. 2. Among which point 1, point 2 and point 3 is the midpoint of line 1, line 2 and line 5, respectively. And the mid-point of the first scanning path of the second layer is marked as point 4 .

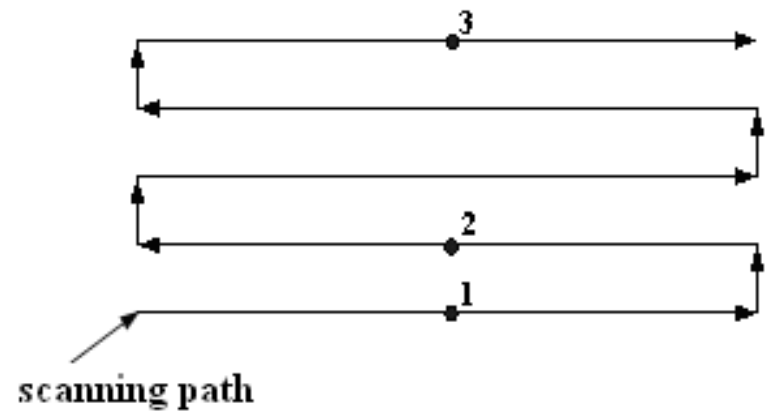

Fig. 2. Scanning pattern of laser sintering

Fig. 3 shows the temperature distribution at time $0.0125 \mathrm{~S}, 0.025 \mathrm{~S}, 0.0375 \mathrm{~S}$. As seen from Fig. 3, With the moving of laser beam the temperature field distribution is different from distribution of rotundity temperature field of stationary laser beam. The concrete expression is that the isotherm of the sintered front-end is closer than that of the sintered area, namely, temperature gradient of the sintered front-end is greater than that of sintered area. The reason is that with the rapidly moving of the highenergy laser beam the heat of laser center spreads around during the sintering process. While the density and thermal conductivity of sintering area are higher than them of powder front-end, thus heat is more easily to spread backward and makes the distance of isotherm of the sintered area enlarge and the temperature distribution exhibit "comet" shape. The simulation results show that zone affected by heat expands constantly and the temperature of substrate and powder bed has increased to some extent as the sintering goes on. This is mainly due to the accumulation of heat during sintering process. Fig. 3 shows that the temperatures of molten pool have exceeded the melting point of $\mathrm{Ni}(1728 \mathrm{~K})$ while the maximum temperatures of molten pool fail to reach the melting point of $\mathrm{W}(3650 \mathrm{~K})$. Therefore, Ni powder has change into liquid while the $\mathrm{W}$ powder keeps on solid phase. It has ensured that the molten pool formed by solid-liquid mixture state. The solid particles of the molten pool dissolved in the liquid phase rearrange, move closer to each other and bond into the frame by the role of liquid surface tension. The remaining liquid evenly distributes in the gap of the skeleton, which avoids effectively the "balling" phenomenon of sintering in pure liquid phase. 


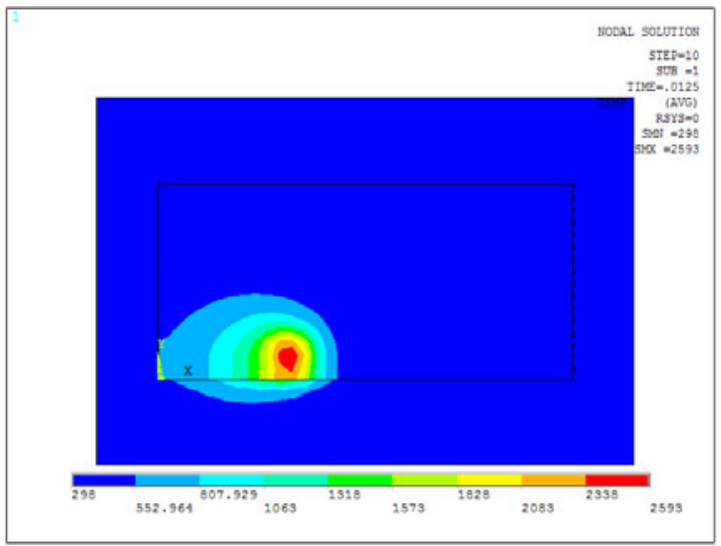

(a) at $0.0125 \mathrm{~S}$.

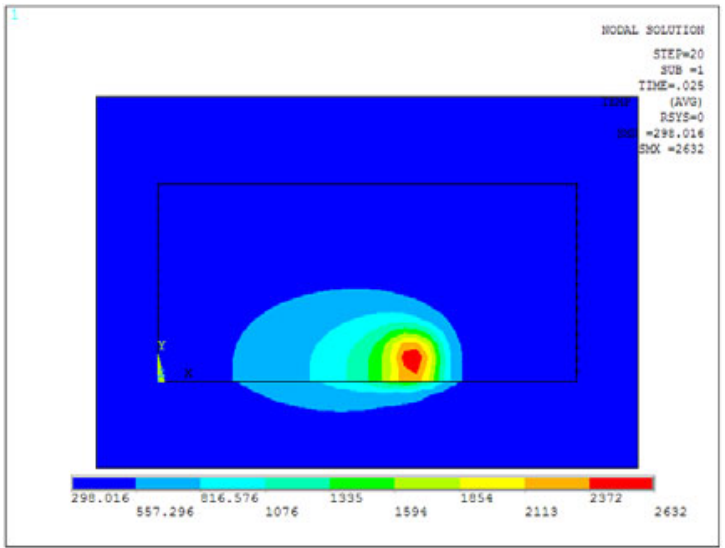

(b) at $0.025 \mathrm{~S}$.

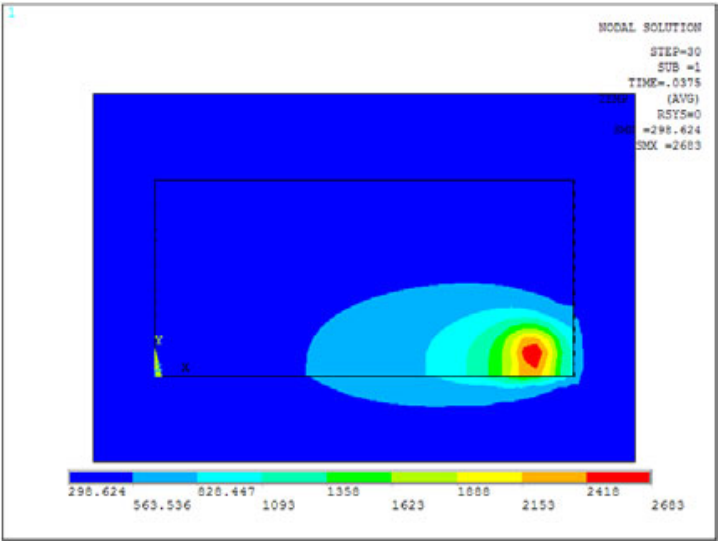

(c) at $0.0375 \mathrm{~S}$.

Fig. 3. Temperature distribution at different moment 
Fig. 4 is the temperatures of point 1 , point 2 and point 3 vary with time. When point 1 is sintering, its temperature rises rapidly to $2628 \mathrm{~K}$ and exceed the melting point of $\mathrm{Ni}$, so the $\mathrm{Ni}$ powder melts. Its temperature gradually reduces when the spot's far away and there's a similar fluctuation with the increase of scanning lines. For sintering two layers and each layer with five scanning, temperature of every point has about 10 similar fluctuations. Moreover in each layer, with the distance between scanning path and point 1 increasing, fluctuation gradually decline, namely, the effect on the temperature of point 1 decrease gradually. In the meantime the highest temperature of subsequent scanning path is little higher than that of previous scanning path, and it has the similar regularity to other points. Fig. 4 shows that the temperatures of point 1, point 2 and point 3 show upward trends throughout all the sintering process, which is due to heat accumulation caused by sustained action of laser beam.

Fig. 5 is the temperatures of point 1 and point 4 vary with time. Point 4 is the midpoint of the first sintering path of the second layer. While sintering the first layer, the element of point 4 has been killed, so the temperature of point 4 is still the initial temperature $(298 \mathrm{~K})$ at the time $0.19375 \mathrm{~S}$. While sintering the second layer, the temperature of point 4 rises gradually. when the laser beam scans through point 4 , the temperature of point 4 sharply rises to near $3000 \mathrm{~K}$ and the temperature of point 1 , under point 4 , correspondingly rises, which is lower than point 4 because of separating a sintering layer and higher than melting point of $\mathrm{Ni}$. The sintering depth is more than $0.1 \mathrm{~mm}$, which shows that these process parameters ensure the metallurgical bonding between layers as well as the bonding strength between layers of sintered parts.

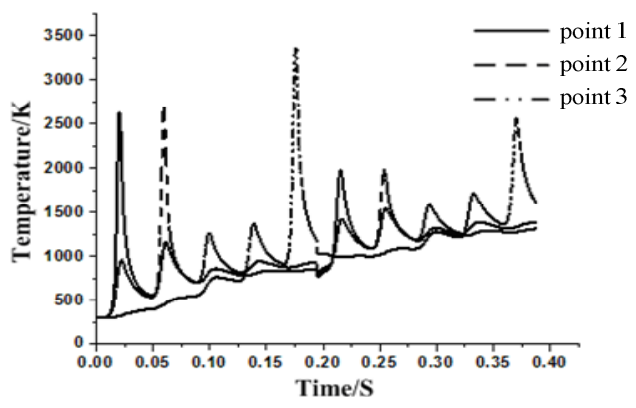

Fig. 4. Temperatures of point 1, point 2 and point 3 varying with time

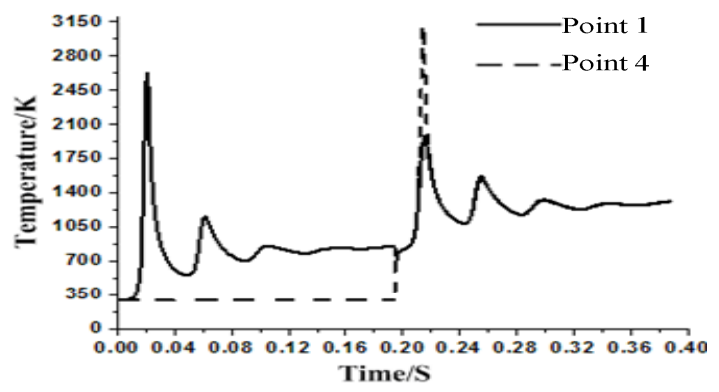

Fig. 5. Temperatures of point 1 and point 4 varying with time 
Fig. 6 is the temperature distribution of Y-direction and Z-direction of point 1 and point 2. From Fig. 6(a), we could see the width of point 2, above the melting point of Ni powder $(1728 \mathrm{~K})$, is about $0.425 \mathrm{~mm}$, which is larger than sintering width $(0.3 \mathrm{~mm})$ and ensures the metallurgical bonding between scan paths. Fig. 6(b) shows that the temperature has decreased with the increase of depth of sintering, the temperature at $0.1 \mathrm{~mm}$ has reached the melting point of Ni powder, which shows that the powder of sintering layer could be melt completely and makes sintering layer and the substrate are bonding firmly. The temperature distribution of point 1 is similar to that of point 2, except for the temperature of point 2 is little higher than point 1 because of the heat accumulation. Compared Fig. 6(a) with Fig. 6(b), we can find that temperature gradient of Y-direction is larger than that of Z-direction, hence, the heat lose is mainly along Z-direction. So it is necessary to find out the influencing factors of the depth of molten pool, which are adjusted to stabilize the molten pool and improve the precision of parts.

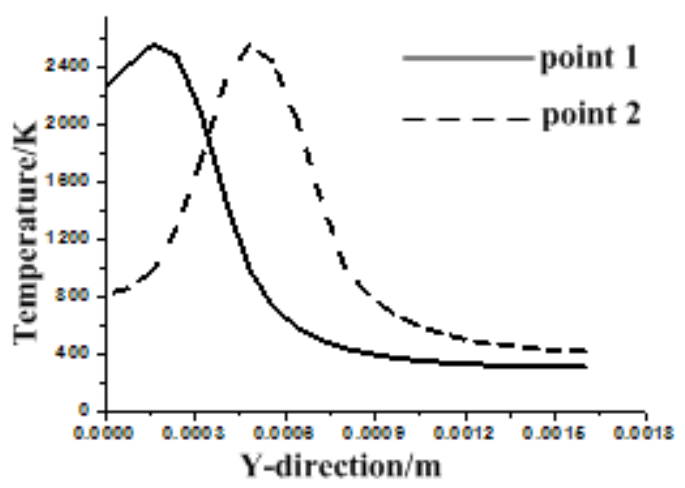

(a) Temperature distribution of Y-direction.

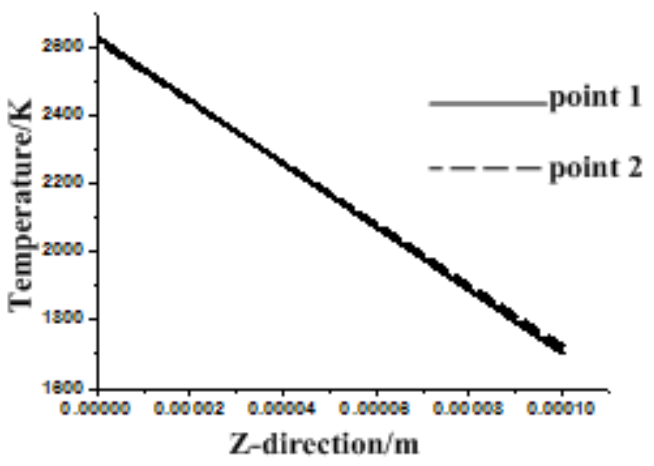

(b) Temperature distribution of Z-direction.

Fig. 6. Temperature distribution of point 1 and point 2 


\section{Conclusion}

Considering comprehensively the influence of temperature-related thermal physical properties, Gaussian laser beam, thermal conduction and heat convection etc., a finite element model of SLS on the two-component metal powder has been developed using APDL and the temperature field has been simulated effectively using the element "birth and death" technology and the dynamical loading of the moving laser beam, which could dynamically reflect the form and change of temperature field during sintering process. The results show that the highest temperature during sintering process is higher than the melting point of Ni powder, but not reaches the melting point of $\mathrm{W}$ powder, so the sintering mechanism is an incomplete molten mechanism, Which is also consistent with forming mechanism of SLS powder mixture. The heat accumulation makes the area affected by heat expand and the whole temperature of substrate and powder bed rise as sintering goes on. It's reasonable to select SLS process parameters according to the width and depth of the molten pool.

\section{Acknowledgements}

We are thankful that the study is supported by Natural Science Foundation of Jiangxi Province of China(2008GZC0055) and Foundation of Jiangxi Educational Committee(GJJ09209).

\section{References}

1. Wang, R.J., Li, X.H., Wu, Q.D.: Optimizing process parameters for selective laser sintering based on neural network and genetic algorithm. Int. J. Adv. Manuf. Technol. 42, 10351042 (2009)

2. Xiong, B.W., Xu, Z.F., Yan, Q.S., et al.: Progress on metal powder materials used for direct selective laser sintering. Hot Working Technology 33(9), 92-94 (2008) (in Chinese)

3. Cui, Y.J., Bai, P.K., Wang, J.H., et al.: The present application and materials for selective laser sintering. New Technology \& New Process 4, 74-77 (2009) (in Chinese)

4. Kolossov, S., Boillat, E., Glardon, R., et al.: 3D FE simulation for temperature evolution in the selective laser sintering process. International Journal of Machine Tools \& Manufacture 44, 117-123 (2004)

5. Guo, H.F., Zhou, J.Z., Zeng, Z.R.: 3D finite simulation for temperature field of direct metal laser sintering. Tool Engineering 40(1), 13-18 (2006) (in Chinese)

6. Zhou, J.Z., Guo, H.F., Xu, D.P., et al.: Finite element simulation for the temperature field in multi-layer thin-wall metal part formed by DMLS. China Mechanical Engineering 18(21), 2618-2623 (2007) (in Chinese)

7. Gu, D.D., Shen, Y.F., Liu, M.C., et al.: Numerical simulations of temperature field in direct metal laser sintering process. Transactions of Nanjing University of Aeronautics \& Astronautics 21(3), 225-233 (2004)

8. Weng, M.C., Yang, Z.Q., Guo, H.F., et al.: Effect of process parameters on the temperature field evolution of Fe Powder laser sintering. Laser Journal 28(3), 70-72 (2007) (in Chinese) 
9. Yang, J., Wang, F.: 3D finite element temperature field modeling for direct laser fabrication. Int. J. Adv. Manuf. Technol. 43, 1060-1068 (2009)

10. Tan, Z., Guo, G.W.: Thermal physical properties of engineering alloys. Chinese Standards Press, Beijing (1988) (in Chinese)

11. Shen, Y.F., Gu, D.D., Li, S.W., et al.: Simulation of 3D transient temperature field during selective laser sintering of multi-component metal powder. Journal of Nanjing University of Aeronautics \& Astronautics 40(5), 611-615 (2008) (in Chinese) 